\title{
An unusual case of DRESS syndrome
}

\author{
Keren Ben-Ari ${ }^{1}$, Ilan Goldberg${ }^{1}$, Idit Shirazi ${ }^{1}$, Iris Amitay², Ma'ayanit Sigler ${ }^{3}$, Sarah Brenner ${ }^{1}$
}

1. Department of Dermatology, Tel Aviv-Sourasky Medical Center and Sackler Faculty of Medicine, Tel Aviv University, Israel

2. Department of Dermatology, Rabin Medical Center, Beilinson Campus, Petah Tiqva, Israel

3. Geha Mental Health Center, Felsenstein Medical Research Center, Rabin Medical Center, Beilinson Campus, PetachTikva and Sackler Faculty of Medicine, Tel Aviv University, Israel

Corresponding author:

Sarah Brenner, MD

Department of Dermatology

Tel Aviv Sourasky Medical Center

6 Weizman Street

Tel Aviv 64239 Israel

E-mail: i-f-skin@smile.net.il

Tel.: 972-3-6973356

Fax: 972-3-6974810

Key words:

drug eruptions, drug hypersensitivity, eosinophilia, paracetamol, phenytoin, schizophrenia

\section{Abstract}

Background: DRESS syndrome (drug reaction with eosinophilia and systemic symptoms) is a rare but severe drug reaction.

Observations: A 27-year-old male with paranoid schizophrenia was hospitalized with all three diagnostic criteria of DRESS syndrome: cutaneous drug eruption, hematological abnormalities (presence of atypical lymphocytes on blood smear) and systemic involvement (generalized lymphadenopathy and hepatitis). On hospitalization the patient exhibited an unusual fever pattern of high temperatures in the morning hours and lower temperatures towards evening. In this case of a patient who needs life-long therapy, we demonstrated the value of the IFN-gamma release test, which showed positive reactivity to 3 out of 9 suspicious drugs: paracetamol, phenytoin and dypirone, allowing for more therapeutic options. After therapy, at 6-month followup the patient is doing well under haloperidol treatment, laboratory values including liver function tests are normal and his skin condition is good.

Conclusion: We suggest that clinicians take the fever pattern of high temperatures in the morning hours and lower temperatures towards evening into account in a patient presenting with a severe cutaneous drug eruption. An interferone-gamma release test may facilitate identification of drugs responsible for the drug reaction.

\section{Introduction}

Skin is the most frequent target of drug reactions. Most, probably more than $90 \%$ of cases, are related to drug hypersensitivity. The clinical presentation of "drug eruptions" is highly variable, from the most common transient and benign erythema that occurs 6-9 days after the introduction of a new drug in 1 to $3 \%$ of users, to the most severe forms (toxic epidermal necrolysis, Steven Johnson syndrome) that affect less than 1/10,000 users. ${ }^{1}$ Hypersensitivity syndrome (HSS) was initially called the anticonvulsant HSS, 2 but reports of similar reactions with several different types of drugs necessitated another denomination. A more informative, precise and clinically relevant term has been proposed: DRESS (Drug Rash with Eosinophilia and Systemic Symptoms). ${ }^{3}$ In its complete form, DRESS syndrome includes a severe eruption, fever, hematologic abnormalities with eosinophilia and/or atypical lymphocytosis, multi-visceral involvement and/or generalized lymphadenopathy. The multi-visceral involvement differentiates DRESS from common cutaneous drug eruptions. ${ }^{3}$ DRESS syndrome occurs in only a relatively small proportion of patients but is associated with considerable morbidity and mortality, which is $10 \%$ if unrecognized and untreated. ${ }^{4}$ DRESS syndrome usually occurs on first exposure to the associated medication with a delayed onset.

We present a patient who met all three diagnostic criteria of DRESS: cutaneous drug eruption, hematological abnormalities (presence of atypical lymphocytes on blood smear) and systemic involvement (generalized lymphadenopathy and hepatitis). Our patient exhibited an unusual pattern of fever that, in combination with adequate symptoms and settings, might be another diagnostic clue to the DRESS syndrome. 


\section{Case Report}

A 27-year-old male, known to have paranoid schizophrenia and being treated with neuroleptics, was admitted to the dermatology department with a diffuse maculopapular exanthema and highly infiltrated skin. The rash was localized first on the breast and trunk with consecutive generalization (Fig. 1). Nikolski sign was negative. The patient exhibited widespread lymphadenopathy, facial edema, severe enteritis, chills, and an unusual fever pattern with high temperatures in the morning and lower temperatures towards evening. This reversal of the normal diurnal pattern is synonymous with the "typhus inversus" pattern. Blood cultures were sterile. The patient was treated with clozapin 10 months prior to admission and phenytoin 6 weeks prior to admission because of few focal epileptic seizures. He admitted to using "hagigat", a drug, recently banned in Israel, made from kat plant extracts whose active ingredient is cathionine (a compound structurally and functionally similar to amphetamine that releases catecholamines from presynaptic storage site).

Abnormal laboratory values included high white blood cell count - 43.3×103/ml (normal 4.0-10.0 ml), without eosinophilia. Liver function tests: GPT (ALAT) - 325U/I (normal 5-39), GOT (ASAT) - 268U/I (normal 7-40), alkaline phosphatase - 217U/l (normal, 60-170), LDH 2269U/I (normal, 25-100) INR - 1.48, albumin - 17g/l and serum total protein - $43.3 \mathrm{~g} / \mathrm{l}$, proteinuria - $435 \mathrm{mg} / 24$ hours. Creatinine level and chest radiography were normal. Serologic tests for viral infections, including cytomegalovirus, Epstein-Barr virus, adenovirus, enterovirus, parvovirus Coxsackievirus, human immunodeficiency virus, hepatitis A, B, and C virus were all negative. Throat swab cultures were repeatedly negative.

Abdominal ultrasound revealed bilateral pleural effusion, hepatosplenomegaly, and a small amount of ascitic fluid. Blood smears showed atypical lymphcytosis. Histological investigation of a skin biopsy showed findings typical of DRESS / drug hypersensitivity syndrome.

The initial differential diagnosis was drug hypersensitivity syndrome versus neuroleptic malignant syndrome (NMS) with cutaneous manifestation, but NMS was ruled out because of absence of muscle rigidity (part of the DSM-IV major criteria). Phenytoin was discontinued immediately and switched to valproic acid. Clozapin was reduced from $400 \mathrm{mg} / \mathrm{d}$ (a pro-convulsive dosage) to 300 $\mathrm{mg} / \mathrm{d}$, and prednisone $30 \mathrm{mg} /$ day was introduced. After a week on this regimen there was no significant change in the patient's condition and all medications were stopped, including antipyretics and corticosteroids.

A few days after the initial hospitalization, the peculiar fever pattern observed during the first days of hospitalization changed to a more physiologic fever pattern of being lowest in the morning and highest in the evening. Repeated blood cultures taken at the time the fever pattern changed were positive for Staphylococcous areus. The patient was started on vancomycin and later switched to IV orbenin following sensitivity testing. His skin condition improved but hepatic function deteriorated: GPT

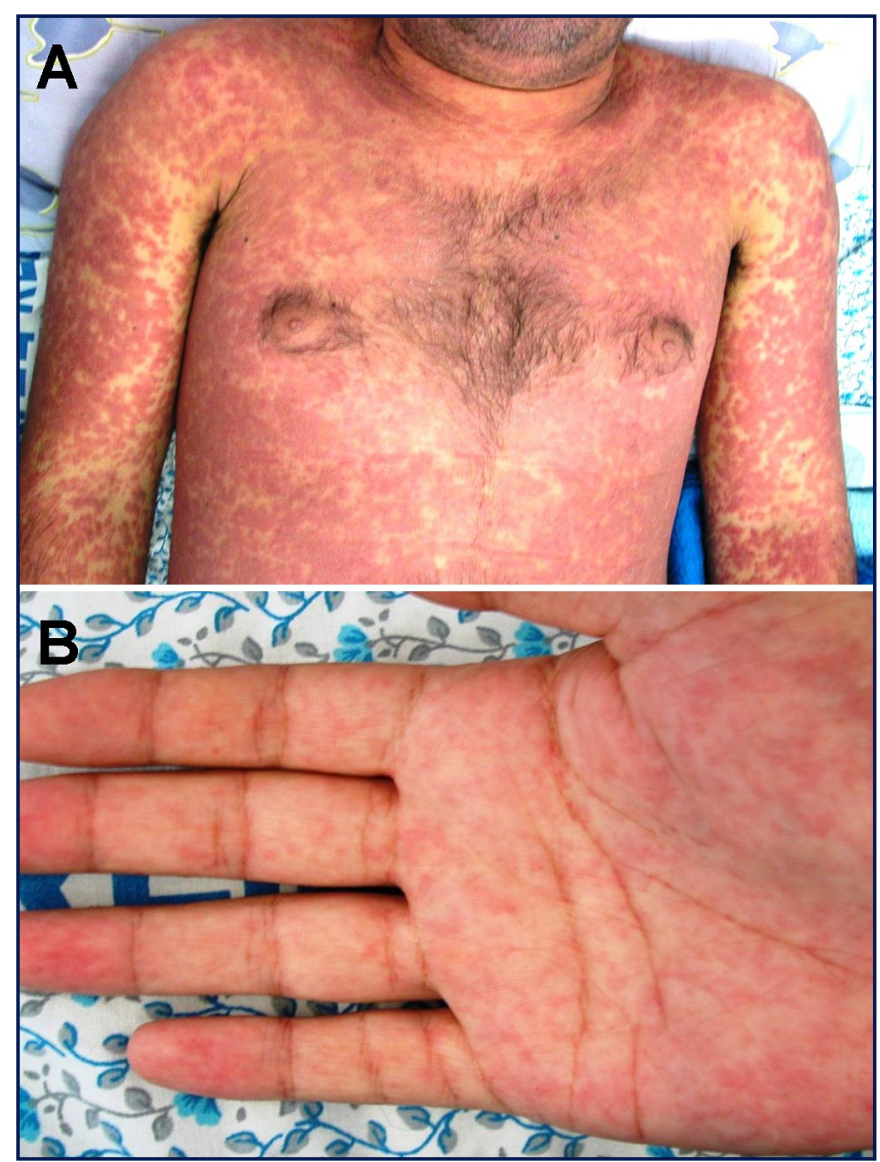

Figure 1

Symmetrically distributed maculo-papular eruption with induration and plaque formation. The eruption later progressed to confluent lesions, erythroderma, and finally to generalized exfoliative dermatitis $(A)$. Fine macular eruption on palms $(B)$ was bilateral.

increased to 1066, albumin decreased to 17 , and INR increased to 2.24. He was transferred to an internal medicine ward where, despite treatment for sepsis with IV antibiotics, he suffered septic shock with unstable blood pressures including an event of blood hemolysis that necessitated blood transfusions. A few days later the fever subsided and liver function tests started to normalize.

Prior to discharge, antipsychotic therapy with quetiapin was initiated; a few days later the patient developed severe facial edema, high fever and new onset of generalized rash, and the quetiapine was stopped immediately. Suspecting the antiepileptic phenytoin to be the cause of his primary DRESS syndrome, clozapine was restarted but a few days later his condition deteriorated further and the clozapine was discontinued again.

Six weeks after admission, following a marked improvement in his condition, the patient was discharged from the hospital and sent to a psychiatric facility for drug adjustment where he was started on levomepromazine and amisulpiride. Four days later the patient was hospitalized in another dermatologic department with renewal of his rash accompanied by systemic symptoms, fever and itching. Levopromazine was discontinued and prednisone was begun. His condition improved and he was 
Table 1

Results of in vitro INF-gamma release test in the patient with DRESS syndrome (during corticosteroid therapy).

\begin{tabular}{|l|l|l|l|}
\hline & $\begin{array}{l}\text { INF-gamma without drug } \\
(\mathrm{pg} / \mathrm{ml})\end{array}$ & $\begin{array}{l}\text { INF-gamma with drug } \\
(\mathrm{pg} / \mathrm{ml})\end{array}$ & $\begin{array}{l}\text { INF-gamma } \\
\text { increase }\end{array}$ \\
\hline Paracetamol & 590 & 766 & 30 \\
Dypirone & 590 & 822 & 39 \\
Phenytoin & 590 & 766 & 30 \\
Clozapine & 590 & 550 & No increase \\
Solian & 590 & 567 & No increase \\
Dekinet & 590 & 580 & No increase \\
Seroquel & 590 & 420 & No increase \\
Nozinan & 590 & 440 & No increase \\
Valproate & 590 & 450 & No increase \\
\hline
\end{tabular}

discharged. At six-month follow-up the patient is doing well under haloperidol treatment, laboratory values including liver function tests are normal and his skin condition is good.

An in vitro IFN-gamma release test was conducted with all 10 suspected drugs (Table 1). The test was carried out twice: once with lymphocytes under corticosteroid suppression and again 3 months after corticosteroid therapy was stopped.

The method has been described in detail elsewhere. 5 Briefly, patients' lymphocytes from heparinized venous blood are separated by Ficoll Hypaque gradient centrifugation and cultured for 24 hours in test tubes containing medium, phytohemagglutinin, and each drug, or medium and phytohemagglutinin without the drug. Unmodified drugs dissolved in the appropriate solvents are used. Following incubation for 24 hours in $5 \% \mathrm{CO} 2$ at $37^{\circ} \mathrm{C}$, the test tubes are centrifuged at $2500 \mathrm{rpm}$ for $25 \mathrm{~min}$ at $5^{\circ} \mathrm{C}$. The supernatants are collected for the detection of INFgamma release using the ELISA technique (Biosource, Enco Diagnostics, Petach Tikvah, Israel). INF-gamma release is reflected in the percentage of its increase calculated by the formula:

$\%$ interferon-gamma increase $=100 \mathrm{X}$ (interferon-gamma with the drug - interferon-gamma with medium alone)/ interferon-gamma with medium alone.

A positive interferon-gamma test response has been determined to be $30 \%$ based on past measurements.

A marked increase of interferon was observed following in vitro challenge of the patient's lymphocytes with dypirone, paracetamol and phenytoin. No significant $(<30 \%)$ increase in IFN was observed with the other drugs tested. It is noteworthy that the depressed response of lymphocytes on the initial test was recorded while the patient was on corticosteroid treatment. As seen from the tables, while there was a slight change in the reactivity of three drugs, there was no change in the specific drugs that reacted.

\section{Discussion}

It is often difficult to determine the causative drug in DRESS. The 'usual suspects' are aromatic anti-epileptics. Numerous drugs have been associated with isolated cutaneous eruptions, in particular of the simple exanthematic or maculopapular type, while only a limited number of drugs are associated with DRESS/HSS, suggesting differences in the pathogenesis of this condition. 6 For phenytoin, the culprit in our case, carbamazepine and phenobarbital, the incidence of DRESS has been estimated to be 1 reaction per 5,000 to 10,000 exposures. ${ }^{6}$

The time lag between the administration of the suspected drug and the onset of DRESS (latent period) is long, usually 2-8 weeks. The latent period between the administration of phenytoin and drug-related symptoms was 6 weeks in our patient, and during that time the patient could also have taken paracetamol and/or dipyrone, implicating all three drugs in the eruption. Based on the literature, phenytoin was the most probable cause. While a single drug is usually considered to be responsible for an adverse drug reaction in a given patient, the role of multiple drugs cannot be ignored. 7

Cross-reactivity was found in our patient to phenytoin, clozapin and quetiapin, but not to other drugs taken by him, including amisulpiride and levopromazine. Phenytoin, clozapin and quetiapin share a common metabolite, arene oxide, which is a well-established factor in DRESS pathogenesis. It may be hypothesized that this common metabolite may explain the undulating pattern of the patient's reaction after each administration of an antipsychotic drug or that this pattern is a part of the natural course of DRESS syndrome.

In this case of polypharmacy in a patient who needs lifelong therapy, we demonstrated the value of the IFN-release test. Suspicion in our case towards 9 drugs was narrowed down to only 3, allowing for more therapeutic options. 
The pattern of fever our patient was unusual, with high temperatures in the morning and low to normal in the evening. Bennet and Hook described such a pattern in 1957 and called it "typhus inversus" fever pattern. ${ }^{8}$ Normal 24-hour circadian temperatures vary typically by 0.5 $1.0^{\circ} \mathrm{C}$ between the A.M. nadir and the P.M. peak. 9,10 In typhus inversus pattern, there is a reversal of the normal diurnal pattern such that the highest temperature peak occurs in the early A.M. hours and the lowest in the evening hours. This type of fever curve may be observed in tuberculosis, especially of the miliary type, hepatic abscess, salmonella bacteremia, endocarditis and rarely has other causes. ${ }^{8}$

It is important to note that this "typhus inversus" fever pattern occurred at the beginning of the hospitalization when blood cultures were sterile, and only when the fever pattern returned to normal diurnal pattern did the blood cultures reveal staphylococcus bacteria. Thus, the typhus inversus fever pattern can not be attributed to the bacteremia encountered later. This sequence of events led us to speculate about the possible association of the typhus inversus fever pattern and DRESS syndrome. It has to be taken into consideration whether this fever pattern be could be another symptom of DRESS syndrome. This fever pattern has to be taken into account as a possible symptom of DRESS syndrome in a patient presenting with a severe cutaneous drug eruption.

\section{References}

1. Roujeau JC. Clinical heterogeneity of drug hypersensitivity. Toxicology. 2005; 209: 123-129.

2. Krivoy N, Taer M, Neuman MG. Antiepileptic drug-induced hypersensitivity syndrome reactions. Curr Drug Saf. 2006; 1: 289-299.

3. Valeyrie-Allanore L, Sassolas B, Roujeau JC. Drug-induced skin, nail and hair disorders. Drug Saf. 2007; 30: 1011-1030.

4. Fitzpatrick's Color Atlas \& Synopsis of Clinical Dermatology. Klaus Wolff, Richard Allen Johnson, Dick Suurmond, Editors. 4th ed. McGraw Hill; 2001. p. 564-565.

5. Yoshimura T, Hamaguchi E, Usami E, Nakashima K, Kawaguchi M, Suzuki N, Okamoto Y, Nakao T, Yamazaki F. Increased in vitro release of interferon-gamma from ampicillin-stimulated peripheral blood mononuclear cells in Stevens-Johnson syndrome. Biol Pharm Bull. 2004; 27: 929-931.

6. Sullivan JR, Shear NH. The drug hypersensitivity syndrome: what is the pathogenesis? Arch Dermatol. 2001; 134: 357-358.

7. Tas S, Simonart T. Management of drug rash with eosinophilia and systemic symptoms (DRESS syndrome): an update. Dermatology. 2003; 206: 353-356.

8. Gaeta GB, Fusco FM, Nardiello S. Fever of unknown origin: a systematic review of the literature for 1995-2004. Nucl Med Commun. 2006; 27: 205-211.

9. Mackowiak PA. The febrile patient: diagnostic, prognostic and therapeutic considerations. Front Biosci. 2004; 9: 2297-2301.

10. Hughey LC. Fever and erythema in the emergency room. Semin Cutan Med Surg. 2007; 26: 133-138. 